\title{
Observing User Interface Design Patterns for Websites from a User-experience Point-of-View
}

\author{
Ashwini G. Varma ${ }^{1}$, Prof. Jignyasa B. Sanghavi ${ }^{2}$ \\ ${ }^{1}$ Dept. of Comp. Science \& Engg. Shri Ramdeobaba College of Engineering \& Management, Nagpur \\ varmaag@rknec.edu \\ ${ }^{2}$ Dept. of Comp. Science \& Engg. Shri Ramdeobaba College of Engineering \& Management, Nagpur \\ sanghavijb@rknec.edu
}

\begin{abstract}
Nowadays, user dashboards have been widely used everywhere, and the design complexity is growing with the more and more advanced technology. A very important aspect in user dashboards design is their specifications that describe what the systems have to do. The specification should provide criteria for the systems to be designed. The criteria are formulated in a specification language, which is kept preferably on a high abstraction level and, for example, need not be executable. The specification gives a formal model by class-library extensions, by this way to ensure the consistency of the systems and define the properties of the systems. In many modern applications, graphs play important role in representing the specifications of user dashboards. The richness and practical purposes are needed by the specifications. The richness comes from several resources, which have the variations in the way of different types of graphs. Besides the general graph, there are Conditional Process Graph (CPG), Petri nets and other types of graphs. It turns more difficult to work on these graph representations in a textual format since now the user dashboards become more and more complicated. We need to develop a visual dashboard, which users can design and edit the graph visually based on certain form of specifications. There exist some kind of dashboards for graphs, but they each mostly focus on one type of graph at a time. If new graph is introduced, they cannot work anymore. So, we propose an extensible visual dashboard, which can edit many types of graph with their own specifications that are defined in formal ways. All the representations in the dashboard are graph-based and they share common concepts such like edges and nodes. By this way, the dashboard becomes extensible and it is not simply the combination of several dashboards. In this paper, we study different methods to design user dashboards, and evaluate the best practices in designing the same.
\end{abstract}

Key words: User, dashboard, user experience, aggregation

\section{INTRODUCTION}

According to a recent study by the University of Texas Center for Research in Electronic Commerce, the US Internet economy generated revenue of 301 billion dollars and was responsible for more than 1.2 million jobs in 2018. The Internet economy has already surpassed traditional sectors such as energy (223 billion) and telecommunications (270 billion) and is comparable with the automobile sector (350 billion). The growth of e-panels activities has been phenomenal and is expected to increase even further. Therefore, the performance measurement for e-panels becomes important with the gradual maturing of the digital market. It also singles out by the fact that the e-retail is going through a crucial period of improvement. Most of the companies need to review their strategies and restructure their operations with trading partners in order to become profitable and viable in the highly competitive e-panels market. E-retailers want to assess the status of their current website, look for improvement and plan for future strategy. Therefore, an accurate control of e-panels Website's performance is needed in order to make E-retailers profitable [1].The Web based application, the E-panels Dashboard, will be developed to provide a means of visualizing and monitoring the performance of the e-panels Website. The E-panels Dashboard is an application, which generates pages in HTML format to provide relevant information about the sales performance of the e-panels Website.

User dashboards are now everywhere applied at present with more and more technologies in design, but the difficulties are increasing at the same time. As an important part of the user dashboards design, the system specifications need to be created as a start. The specification is a behavioural description of the system and specifies the requirements for a system, and it includes both functional requirements, the operations to be performed by the system, and non-functional requirements such as speed, power and design cost. The specifications should be implementation-independent, that is to say, the specification is composed without any implications about the structure of the implementation [2].

The system is called 'correct' if the criteria specified inside the specification are satisfied. These criteria are formulated in a specification language, which is kept preferably on a high abstraction level and, for example, need not be executable. The specification language should support the creative development of the specifications. Formal or informal specification languages can be used by the designer to specify the intended behaviour of the system and mapped into the system model later in design flow. Specification models can be sorted into 
different categories by what they mainly focus on. Among them, graph-based representations are the most popular in embedded system design. For instance, Petri nets are a kind of state-oriented specification model; CPG is a kind of activityoriented specification model; and we have many other graph representations like state charts and so on [3].

Since now the systems are getting more and more complicated, it is more difficult to go on with the design if the user dashboards specifications are represented only in textual formats. So, we need to make the user dashboards specifications in a visual way. There have already some graph dashboards being designed for different types of graphs. For example, Predator dashboard and GrapE are especially for Petri nets; and there are variant kinds of dashboards for general graphs, which always have different emphasis in design. For CPG it is good to use project JGraph (Project website: http://jgraph.sourceforge.net/), thus we implement a prototype of the CPG dashboard by generalizing the basis object classes from JGraph later in this paper. But if the users need to edit variant types of the graph at one time, they have to load different visual dashboards. So, it is very practical to extend the graph dashboard due to the user dashboards' design demands [4].

Since almost all the graphs share some common properties and attributes on the nodes, edges and etc. we can provide a new extensible visual dashboard solving the above problem. Even with the new type of graph that we never meet before, because of the similarities in the graph property, we can just base on the original library of the extensible dashboard and extends the corresponding classes to implement a prototype for the new type of graph. Under the instructions of the respective specifications for each kind of graph, through kind of plug-in technology, the dashboard is extensible with displaying different toolbars, menus automatically when loading the graphs [5].To design the extensible visual dashboard, we discuss the software architecture and the structures of the graph-type specification files. In addition, we give the examples of the specification files for general graphs, CPGs and Petri nets each in the paper. And then, one implementation prototype of a dashboard for CPGs is also available with the basic functionality like open, edit and save. The next section describes various user dashboard approaches, followed by their comparative analysis. Finally, we conclude the paper with some acute observations and some future research directions which can be followed by various aggregators.

\section{LITERATURE REVIEW}

In order to identify the techniques of building an effective interface design, In [6], researcher asked the following question, "How can we think about it so that the interfaces we design will empower users?" "What does the user want to do?" By answering these questions, Laurel suggested that designers should keep in mind that design might focus less on the technology and more on the user. Working with this perspective, computer interfaces should be designed so that the user interacts more with the task and less with the machine.
She believed in "making computers more powerful extensions of our natural capabilities and goals" by offering the user a familiar environment. One way of achieving this goal is through creative use of colour. Colour can not only impart information but it can also be a useful mnemonic device to create associations. Therefore, a colour scheme should be developed for the E-panels Dashboard to provide an accountable and effortless interface for the user. For example, warnings with thresholds of certain parameters are to be shown is different colour to catch the user's attention [7].

Another point mentioned in the article to provide the user with familiar elements is the use of metaphor. A good metaphor is essential to an easy-to-use human interface because it allows us to take our knowledge of familiar objects or events and use it to give structure to abstract and less well understood concepts. Laurel saw metaphors in interface design as a mean to allow people to understand and predict system behaviour. Metaphors can be also used as a convenient means of portraying system attributes and operational tools in a way that does not require technical knowledge. Metaphors are ubiquitous in computer use: "We place windows on our desktop, the put folders within the windows thus forming a tree." Consequently, a metaphor should be chosen for the E-panels Dashboard [8]. The dashboard should have Web-surfing-like user interface just like a dashboard to provide the user with a familiar workplace.

As computer programs increase in complexity, there is a risk that they are becoming harder to use. This ease of use of complex program is made possible by the interface, which presents a facade or illusion of simplicity, which shows that the importance of an interface design can affect the system performance. While computer design may still be the exclusive domain of the engineer or scientist, interface design requires imaginative and creative solutions that cannot be inspired by science alone. Therefore, the designer of the interface should always keep in mind that the design should be user friendly [10],[21]. There are many ways of achieving an interface design that reflects the constraints of the working environment. There is a method called as the interface from a method Work Domain Analysis. It provides a systematic way of determining constraints in the environment that can be used for design. The second part is to convert those constraints into a perceptually usable design by deriving information requirements from the work domain analysis. In order to incorporate domain information into the design, developers need a systematic method for searching for this information, asking the right kinds of questions, and storing these relationships in a way that they can use later. They need to know that they have covered different kinds of constraints and have achieved an adequate breadth of coverage. A framework that is proposed for understanding work domain constraints is termed as Abstraction Hierarchy [11]. A five-level abstraction hierarchy model will be used, where all the constraint of the E-panels Dashboard would be listed. Abstraction hierarchy consists of five different levels, which demonstrates the properties of the system. These five levels are functional purpose, abstract function, generalized function, physical functions, and physical forms. At each level, it demonstrates different characteristics of the work domain. 
Before going into the work domain analysis, a brief description of the work domain and the system boundary will be given. The E-panels Dashboard is to display the Key Performance indicators (KPIs) of an e-panels Website [12]. It provides information to the users and warns the users if any abnormal states occurred. The dashboard would be used by users who do not have a strong technical background. The system boundary is defined as monitoring the e-panels Website of the FTD.com. Only business performance measurement would be taken into account. Technical figures such as transaction rate of the network would not be included in the E-panels Dashboard [13]. The following process is used in order to design the UI/UX for the users,

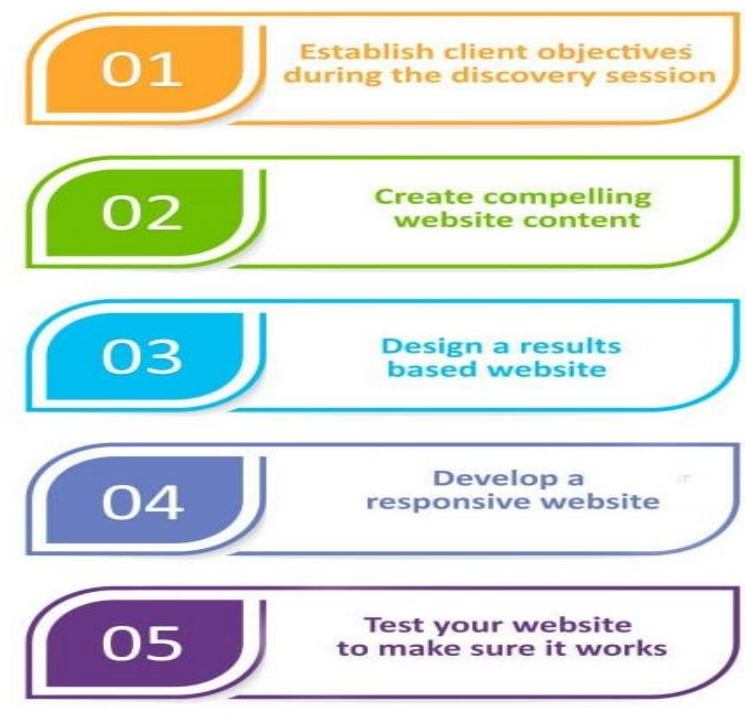

Figure 1: Design process

Functional purpose basically determines what the work domain was designed to do. In this case, the objective of User panel Dashboard will be shown in the function purpose levels. The two objectives of the User panel Dashboard are visualizing the website performance, and monitoring the status of the website, which alerts the users if problems occurred. Abstract function is a description of the casual relationships underlying the work domain. This usually refers to laws that cannot be broken and the priorities that must be achieved. The principle from Ecological interface design, Human centred design and emetrics measurement the user panel performance is included in this level [14]. Generalized function level explains how the casual laws of the abstraction level are achieved. In this level, the processes in the system are shown for analysis. In User panel Dashboard, processes like monitor business transactions, information organization, information categorization, data comparison, information display and information requirement are included in this level. Physical functions level usually refers to various components of the work domain and their capabilities. In the User panel Dashboard, it will be the relevant information provided. It will be the data related to Customer, Sales, Product and Historical Data. Physical forms level is a description of the physical appearance of the work domain. This includes different kinds of performance indicators. All the KPIs are included in this level [15].
In methodical design of user dashboards, one key aspect is the creation of models. The models are the concrete representation of knowledge and ideas about a system being developed. The model may deliberately hide or change some details in the system but concretely give certain properties to be understood, analysed and confirmed. By creation a system model, we can obviously deal with the system complexity well. Usually the creation of a system model starts after the specification. It can be considered as a connection between software application and implementation [16][23]. The system modelling hierarchically organizes components in synchronous or asynchronous communications. The purpose of a model is to provide an abstract view of a system. As a model, the qualities are: it should be formal and no ambiguity, it should be complete, comprehensible, easy to model and neutral enough to aid. Models are easy to define, but it is hard to see their eventual effectiveness and limitations. Good models should be simple, executable, synthesizable, and have high expressive power. The model quality is tested in practice. Generally, there are two types of embedded system modelling: system structure and system behaviour. Behaviour modelling tells what the system does. In real time embedded system, behaviour modelling meets temporal contract with the environment. Structure modelling is needed because the user dashboards often have complex high-level run time structures. Structure modelling gives the relationships between individual entities and communication objects. The popular models for user dashboards are communicating finite state machines (FSM), dataflow, process network, Petri nets, object-oriented models, heterogonous models and so on. Many designs in embedded system are usually based on heterogeneous architectures that integrate multiple programmable processes and dedicated components. So, we can use graph as an abstract syntax of the embedded system models with a useful visual representation.

The concepts of the development environment for interactive software applications could further be applied in business. Defining the user interface model, in terms of user interface objects (like windows, icons, identifiable concepts in the user interface) and user dialogue descriptions (which actions can users perform at a certain dialogue state), was e.g. very useful during my long time secondment at IBM's Human Factors department: user interface development was recognized as a separate discipline and further guided by the Common User Access user interface standard as part of IBM's Systems Application Architecture for IBM's software applications, along with their OS/2 user interface environment. In addition to user interface development, these publications illustrated that not only in the development phase, but also in the testing phase of software applications the identification of user interface objects and user dialogue descriptions could be applied, addressing the intuitiveness and efficiency of the user interface. The development and evaluation of user interfaces was first coined as usability engineering. Baan was one of the world-wide best performing ERP-vendors. As development manager 'user interfaces' I was involved in explicitly including user interface design and testing in Baan's software development cycle. Moreover, principles of user interface design were included in Baan's developed graphical user interface style guide ("Baan GUI"). As such, principles of the development environment for 
interactive software applications on user interfaces were included in the Baan development cycle [17],[22].

The concept of aligning software application implementation with organizational strategy, organizational processes and ITstrategy became central. This concept can be labelled business/IT-alignment. Our extension to business/IT-alignment included an explication of human factors in general (and user interfaces particular) when implementing IT. Works like [1015] show particular operationalizations of business/ITalignment in different industry sectors and in different business functions. Although there is a growing interest in dashboard design, only little research has been done so far. And even if done so, only a few were able to show a positive pattern between mobile campaigns and consumer's post intentions. Researchers have been struggling to outline a perfect framework, and ambiguous results are seen in almost all of their work so far [18][23].

One of the first researches made in the field of dashboard design was in [19], who wanted to measure the performance effect of mobile optimized banners compared to standard display banners. They used a website of a local newspaper and showed the users browsing through their mobile devices the two types of banners, measuring their performance by the clickthrough rates received. Final results supported their hypothesis and mobile optimized banners clearly outperformed non-mobile banners. However, further research by the mobile marketing app platform Trademob showed that $40 \%$ of the clicks in dashboard design are driven by the "fat finger effect." A theory that explains clicks that occur once one's fingertips are larger than the place that is intended to touch on the screen. A more recent study on mobile dashboard design gathered data over 54 mobile campaigns in 10 different industries. The authors used a questionnaire to measures the attitudes of users who were exposed to at least one of the campaigns and to those who were not exposed to any of them. Results showed negative significance, stating that the majority of the campaigns in the data set did not receive a positive customer's attitude or post campaign intentions [20].

From a scientific point of view, this paper will build upon the existing literature and bring significant contributions to analysing MDA effectiveness. Unlike any previous research done so far, the user's behaviour is measured based on their exact responses. Using various tracking platforms, the different campaign performance is examined not only based on the clickthrough rates observed, but also on the conversion rate of applications installed. For a conversion to be counted as successful the user just needs to download and install the application from the App Store or Play Store. The applications promoted in this study are free of charge and no payments were required through the process.

Dashboard design has been growing steadily in the past years and predictions forecast that the global mobile ad spend will continue to grow at an average of $26 \%$ each year over the next five years (2013 Business Insider). Its increased popularity is mainly due to the fact that advertisers have unique possibilities to reach users, unlike any other traditional media before. Even comparing mobile to desktop advertising, there are certain characteristics which outline mobile as the more attractive medium to use.

First of all mobile phones are personal devices and the chances for a certain ad to reach its exact target are fairly high. Computers on the other hand are commonly used by all members of the household and the uncertainty of reaching the audience can be higher for advertisers. Secondly, smart phones are small devices which fit in any pocket and are carried almost always by the users regardless of the situation: e.g. being at a concert, shopping or just going out at night. The highly narrowed locational data available allows advertisers to target their audience more precisely. Geo-local promotions or coupons for restaurants and shops can be delivered to potential customers within the area. Thirdly, dashboard design allows advertisers to target users without any time barriers. Traditional media channels like TV gives advertisers the opportunity to influence consumers only during a certain time frame or a specific programme. The wireless nature of phones is a great possibility to overcome those limitations.

Two main factors are considered as possible limitations to dashboard design: low bandwidth (internet speed) and restricted screen size (Bart, Stephen \& Sarvary 2012). The limited physical space mobile ads have as a mean to express advertising message, can be a possible issue for advertiser. The content which is carried over is relatively short and harder for products under high involvement to be advertised. Mobile industry has evolved significantly and respectively the ways of digital advertising have changed as well. In this paper the different types of dashboard design will be discussed briefly but the focus of the research will be turned on dashboard design.

\section{CONCLUSION}

From the review we can conclude that the overall dashboard design depends on multiple factors like the target audience, the number of options to be displayed on the frontal screen, etc. Thereby using bold designs and combing them with more screen display by reducing unwanted screen elements is the way to go for dashboard design. Moreover, use of graphical visualization tools like Petri-nets will always be beneficial to the user's experience. In order to make a good dashboard, the designer must keep a track of the usability and the number of options which are displayed to the user on the dashboard.

\section{FUTURE WORK}

Due to the emerging nature of block chain based techniques, researchers can try to integrated block chain into the proposed user paneland observe the result improvements in user's authentication performance and removal along with the overall security of the website. This will help the researchers to further study the effects of block chain in user management and explore more areas in the field of application. Moreover, machine learning can be integrated to check which options the user is using frequently and then presenting those options on the dashboard, while keeping other options in their respective hierarchy can be a better way to design user interfaces. 


\section{REFERENCES}

[1] A. Oulasvirta, "User Interface Design with Combinatorial Optimization", in Computer, vol. 50, no. 1, pp. 40-47, Jan. 2017. https://doi.org/10.1109/MC.2017.6

[2] M. D. Harrison, P. Masci, J. C. Campos and P. Curzon, "Verification of User Interface Software: The Example of Use-Related Safety Requirements and Programmable Medical Devices", in IEEE Transactions on Human-Machine Systems, vol. 47, no. 6, pp. 834-846, Dec. 2017.

[3] J. Y. C. Chen, E. C. Haas and M. J. Barnes, "Human Performance Issues and User Interface Design for Teleoperated Robots", in IEEE Transactions on Systems, Man, and Cybernetics, Part C (Applications and Reviews), vol. 37, no. 6, pp. 1231-1245, Nov. 2007. https://doi.org/10.1109/TSMCC.2007.905819

[4] Roth, Robert. (2017) "User Interface and User Experience (UI/UX) Design", Geographic Information Science \& Technology Body of Knowledge. 2017. 10.22224/gistbok/2017.2.5.

[5] Chen, Jessie \& Barnes, Michael \& Harper-Sciarini, Michelle. (2011) "Supervisory Control of Multiple Robots: Human-Performance Issues and User-Interface Design", in IEEE Transactions on Systems, Man, and Cybernetics, Part C. 41 . 435-454. 10.1109/TSMCC.2010.2056682.

[6] M. D. Harrison, P. Masci and J. C. Campos, "Verification Templates for the Analysis of User Interface Software Design", in IEEE Transactions on Software Engineering, vol. 45, no. 8, pp. 802-822, 1 Aug. 2019. doi: 10.1109/TSE.2018.280493

[7] C. Baber, N. S. Morar and F. McCabe, "Ecological Interface Design, the Proximity Compatibility Principle, and Automation Reliability in Road Traffic Management", in IEEE Transactions on Human-Machine Systems, vol. 49, no. 3, pp. 241-249, June 2019

[8] T. Sapounidis, D. Stamovlasis and S. Demetriadis, "Latent Class Modeling of Children's Preference Profiles on Tangible and Graphical Robot Programming," in IEEE Transactions on Education, vol. 62, no. 2, pp. 127-133, May 2019. https://doi.org/10.1109/TE.2018.2876363

[9] J. Y. C. Chen, E. C. Haas and M. J. Barnes, "Human Performance Issues and User Interface Design for Teleoperated Robots", in IEEE Transactions on Systems, Man, and Cybernetics, Part C (Applications and Reviews), vol. 37, no. 6, pp. 1231-1245, Nov. 2007.

[10] Balazs Kovacs, Peter O’Donovan, Kavita Bala, 'ContextAware Asset Search for Graphic Design", IEEE Transactions on Visualization and Computer Graphics, 2019

[11] K. Vallerio, L. Zhong and N. Jha, "Energy-Efficient Graphical User Interface Design", in IEEE Transactions on Mobile Computing, vol. 5, no. 07, pp. 846-859, 2006. https://doi.org/10.1109/TMC.2006.97

[12]R. R. Penner and E. S. Steinmetz, "Model-based automation of the design of user interfaces to digital control systems", in IEEE Transactions on Systems, Man, and Cybernetics - Part A: Systems and Humans, vol. 32, no. 1, pp. 41-49, Jan. 2002.

[13]B. A. Schwendimann et al., "Perceiving Learning at a Glance: A Systematic Literature Review of Learning Dashboard Research", in IEEE Transactions on Learning Technologies, vol. 10, no. 1, pp. 30-41, 1 Jan.-March 2017.

[14]R. Bodily and K. Verbert, "Review of Research on Student-Facing Learning Analytics Dashboards and Educational Recommender Systems", in IEEE Transactions on Learning Technologies, vol. 10, no. 4, pp. 405-418, 1 Oct.-Dec. 2017.

https://doi.org/10.1109/TLT.2017.2740172

[15] S. Charleer, A. V. Moere, J. Klerkx, K. Verbert and T. De Laet, "Learning Analytics Dashboards to Support Adviser-Student Dialogue", in IEEE Transactions on Learning Technologies, vol. 11, no. 3, pp. 389-399, 1 JulySept. 2018.

[16]Z. Qu and J. Hullman, "Keeping Multiple Views Consistent: Constraints, Validations, and Exceptions in Visualization Authoring", in IEEE Transactions on Visualization and Computer Graphics, vol. 24, no. 1, pp. 468-477, Jan. 2018.

https://doi.org/10.1109/TVCG.2017.2744198

[17] Nafar, F., Shamsi, H. "On the Design of a User Interface for an RFID-Based Vehicle Tracking System", Int J Wireless Inf Networks 24, 56-61 (2017). https://doi.org/10.1007/s10776-016-0329-9

[18] Andrea Vázquez-Ingelmo, 'Extending a dashboard meta-model to account for users' characteristics and goals for enhancing personalization”, IEEE, 2018

[19] Andrea Vázquez-Ingelmo, Francisco José García-Peñalvo, and Roberto Therón. 2019 "Tailored information dashboards: A systematic mapping of the literature", In Proceedings of the XX International Conference on Human Computer Interaction (Interacci\&\#xf3;n '19). Association for Computing Machinery, New York, NY, USA, Article $26,1-8$.

[20] JOHN J. DUDLEY, "A Review of User Interface Design for Interactive Machine Learning”, ACM Transactions on Interactive Intelligent Systems, Vol. 1, No. 1, Article 1. Publication date: March 2018

https://doi.org/10.1145/3185517

[21] Fareeha Tariq, Dr. Lili Marziana Bt. Abdullah, Suriani Bt. Sulaiman, Waqar Tariq, "Energy Saving Monitoring Using Fuzzy Logic for Computing Lab”, in International Journal of Advanced Trends in Computer Science and Engineering, 8(1.6), 2019, 190 - 194

https://doi.org/10.30534/ijatcse/2019/2981.62019

[22] Reem Alnanih, Nadia Bahatheg, Melad Alamri, Rana Algizani "Mobile-D Approach-based Persona for Designing User Interface", in International Journal of Advanced Trends in Computer Science and Engineering Available Volume 8, No.5, September - October 2019 https://doi.org/10.30534/ijatcse/2019/111852019

[23] DipaliChordiy,Pranita Bavaskar, Ashwini Gangurde, Pragati Gorde, Prof. Pankaj Badguja, "Design and Implementation of CANBus like Protocol for Indian Automobile", in International Journal on Recent and Innovation Trends in Computing and Communication ISSN: 2321-8169 Volume: Volume: 5 Issue: 2. 